\title{
Quantitative Measurement of Tetrahydromenaquinone-9 in Cheese Fermented by Propionibacteria
}

\author{
K. Hojo, ${ }^{1}$ R. Watanabe, T. Mori, and N. Taketomo \\ Food Science Institute, Meiji Dairies Corporation, 540 Naruda, Odawara, Kanagawa 250-0862, Japan
}

\begin{abstract}
Propionibacteria produce tetrahydromenaquinone-9 [MK-9 $(4 \mathrm{H})]$ as a major menaquinone (vitamin $\mathrm{K}_{2}$ ). This study aimed to determine the MK-9 $(4 \mathrm{H})$ concentration in commercial propionibacteria-fermented cheese. The MK-9 (4H) concentration was quantified using an HPLC instrument with a fluorescence detector after postcolumn reduction. Among the various cheese samples, the MK-9 $(4 \mathrm{H})$ concentration was highest in Norwegian Jarlsberg cheese, followed by Swiss Emmental cheese. In contrast, the MK-9 $(4 \mathrm{H})$ concentrations in Appenzeller or Gruyère cheeses were extremely low or undetected. Likewise, the concentrations in Comte and Raclette cheeses were lower than those in Jarlsberg and Emmental cheeses. In the present study, the MK$9(4 \mathrm{H})$ concentration in cheese showed a correlation with the viable propionibacterial cell count and propionate concentration. This implies that the increase in propionibacteria contributed to the generation of MK$9(4 \mathrm{H})$ in cheese. We presumed, based on these results, that Swiss Emmental and Norwegian Jarlsberg cheeses contain a meaningful amount of vitamin $\mathrm{K}$ because of their high MK-9 (4H) concentrations (200 to $650 \mathrm{ng} / \mathrm{g}$ ). Key words: vitamin $\mathrm{K}$, menaquinone, propionibacteria, cheese
\end{abstract}

\section{INTRODUCTION}

Vitamin $\mathrm{K}$ exists naturally in 2 forms, namely, vitamin $\mathrm{K}_{1}$ (phylloquinone) in green plants and vitamin $\mathrm{K}_{2}$ (menaquinone) in animals and bacteria. Vitamin $\mathrm{K}$ is an essential cofactor for the formation of $\gamma$-carboxyglutamic acid residues in proteins (Shearer, 1990). The $\gamma$-carboxyglutamic acid-containing proteins bind calcium ions and influence blood coagulation and tissue calcification. Vitamin K deficiency has been implicated in several clinical ailments such as intracranial hemorrhage in newborn infants (Purves, 2005) and possible

Received December 242006.

Accepted May 3, 2007.

${ }^{1}$ Corresponding author: kenichi_houjou@meiji-milk.com bone fracture resulting from osteoporosis (Iwamoto et al., 2004). To decrease the risk of these diseases, therefore, vitamin $\mathrm{K}$ food composition data are required. Further, to appropriately counsel patients taking the antithrombotic medicine warfarin, a vitamin $\mathrm{K}$ antagonist, accurate food composition data are of importance.

Early food composition data regarding vitamin $\mathrm{K}$ used chick bioassays that were more qualitative than quantitative. Recently, the development of reliable chromatographic procedures has resulted in greater sensitivity and accuracy of vitamin $\mathrm{K}$ measurements in foods (Koivu-Tikkanen et al., 2000; Schurgers and Vermeer, 2000). These quantitative analyses have revealed that vitamin $\mathrm{K}_{2}$ occurs in foods in various molecular forms, such as menaquinone (MK)-4 and MK-7. Therefore, more reliable data are needed on the menaquinone contents in foods.

Numerous types of cheeses are made worldwide, and they are generally considered menaquinone-containing dairy products. Menaquinones are of microbial origin and are classified based on the number of isoprene units in their side chains. The components of menaquinones differ among various types of cheeses. For example, Koivu-Tikkanen et al. (2000) reported that the total amount of menaquinones was higher in Edam-type cheeses than in Emmental-type cheeses because of the higher MK-8 and MK-9 concentrations in the former. According to their study, Emmental cheeses contained substantial amounts of only MK-4 and phylloquinone, regardless of the aging time.

Here, we focused attention on the starter cultures of various cheeses. Swiss-type cheese is generally prepared with lactic acid bacteria and propionibacteria, whereas Edam-type cheese is usually prepared with only lactic acid bacteria. Morishita et al. (1999) indicated, based on the determination of menaquinones in lactic acid bacteria, that Lactococcus lactis ssp. lactis and Lc. lactis ssp. cremoris, which are the starter cultures of Edam-type cheese, produce mainly MK-8 and MK-9. This information corresponds with the fact that Edam-type cheese contains large amounts of MK-8 and MK-9. On the other hand, propionibacteria have been reported to produce tetrahydromenaquinone-9 [MK-9 
Table 1. Cheese samples in this study

\begin{tabular}{lll}
\hline Sample ID & Cheese sample & Origin \\
\hline A-1 & Appenzeller & Swiss \\
A-2 & Appenzeller & Swiss \\
C-1 & Comte & France \\
C-2 & Comte & France \\
E-1 & Emmental & Swiss \\
E-2 & Emmental & Swiss \\
G-1 & Gruyère & Swiss \\
G-2 & Gruyère & Swiss \\
J-1 & Jarlsberg & Norway \\
R-1 & Raclette & Swiss \\
\hline
\end{tabular}

(4H)] as a major menaquinone (Schwartz, 1973; Furuichi et al., 2006). Propionibacteria are particular to the Swiss-type cheese Emmental and to Gruyère, French Comte, and Norwegian Jarlsberg cheeses (Bouton et al., 1998; Kure and Skaar, 2000; Thierry et al., 2005). Therefore, measurement of the MK-9 $(4 \mathrm{H})$ concentration in these cheeses is of interest.

Recently, Furuichi et al. (2006) found that the Propionibacterium freudenreichii strain isolated from Swisstype cheese produced a large amount of MK-9 (4H) in milk whey medium. They suggested that the menaquinone-producing ability of $P$. freudenreichii was equivalent to that of Bacillus subtilis, which is used for making natto, a Japanese fermented soybean food. However, to the best of our knowledge, no information is available on the MK-9 (4H) concentrations in various cheeses. This study was aimed at performing quantitative measurements of MK-9 (4H) concentrations in several Swiss-type cheeses. In addition, to examine whether a biomass of propionibacteria was associated with the MK-9 (4H) concentration, we measured the concentrations of short-chain fatty acids and the viable cell counts of lactic acid bacteria and propionibacteria in Swisstype cheeses.

\section{MATERIALS AND METHODS}

\section{Cheese Samples}

Cheese samples were purchased in 2006 from several retail stores in Tokyo, Japan. Only propionibacteriafermented cheese samples were selected. Table 1 shows the types of cheeses used in the study. The samples were vacuum-packed in plastic bags and stored at $4{ }^{\circ} \mathrm{C}$ in the dark until analyzed.

\section{Bacteria}

Propionibacterium freudenreichii ET-3 was used in this study. The strain was isolated from a Swiss-type cheese and stocked in the Food Science Institute of Meiji Dairies, Odawara, Japan.

\section{Chemicals and Reagents}

Vitamin $\mathrm{K}_{1}$ (phylloquinone), MK-4 standards, and all reagents used for vitamin $\mathrm{K}$ determination were of HPLC grade and were purchased from Wako Pure Chemical Industries (Osaka, Japan).

\section{Enumeration of Lactic Acid Bacteria and Propionibacteria}

The cheese samples were diluted with saline $(0.85 \%$ $\mathrm{NaCl}$ ), and a series of 10 -fold dilutions was prepared. The series were plated onto the following 3 selective agar plates: M17 (Difco, Becton Dickinson, Sparks, MD) with $0.5 \%$ (wt/wt) lactose and 1.5\% (wt/wt) agar for streptococci, DeMan, Rogosa, Sharpe (MRS, Difco) for lactobacilli, and yeast-extract lactate (YEL; Britz and Riedel, 1994) for propionibacteria. The M17 and MRS agar plates were incubated aerobically at $37^{\circ} \mathrm{C}$ for 48 h. The YEL agar plates were incubated anaerobically at $30^{\circ} \mathrm{C}$ for $72 \mathrm{~h}$. After incubation, the colonies were counted according to the morphology of each colony, and representatives were checked using the catalase reaction, gram reaction, and cellular morphology. Measurement was performed twice independently, and the results were averaged.

\section{Assays of Short-Chain Fatty Acids}

Short-chain fatty acids were determined with an HPLC system as described by Niwa et al. (2002). Ten microliters of each sample was injected into an HPLC system equipped with an electric conductivity detector (Shimadzu Co., Kyoto, Japan) after removing the proteins by treatment with Carrez-1 solution (53.5 g of $\mathrm{ZnSO}_{4} \cdot 7 \mathrm{H}_{2} \mathrm{O} / 100 \mathrm{~mL}$ of distilled water) and Carrez-2 solution $\left(17.2 \mathrm{~g}\right.$ of $\mathrm{K}_{4}\left[\mathrm{Fe}(\mathrm{CN})_{6}\right] \cdot 3 \mathrm{H}_{2} \mathrm{O} / 100 \mathrm{~mL}$ of distilled water). The proteins in $4 \mathrm{~mL}$ of 10 diluted cheese samples were precipitated by adding $0.5 \mathrm{~mL}$ of Carrez-1 solution and $0.5 \mathrm{~mL}$ of Carrez-2 solution. Measurement was performed twice independently, and the results were averaged.

\section{Preparation of the MK-9 (4H) Standard}

Tetrahydromenaquinone-9 was extracted from $P$. freudenreichii ET-3 according to a procedure previously described by Furuichi et al. (2006). Crude extracts were placed on a silica gel column [Silica SG120 column, $20 \times 250 \mathrm{~mm}$ (Shiseido, Tokyo, Japan); mobile phase, hexane and diethyl ether (100:0.8 vol/vol); temperature, $35^{\circ} \mathrm{C}$; and flow rate, $\left.9.9 \mathrm{~mL} / \mathrm{min}\right]$. The fraction containing MK-9 $(4 \mathrm{H})$ was separated by spectrophotometric monitoring at $254 \mathrm{~nm}$. The putative MK-9 $(4 \mathrm{H})$ peak was manually collected into a test tube. The collected 
fraction was evaporated and then redissolved in a small amount of acetone. This crude solution of MK-9 $(4 \mathrm{H})$ was subsequently purified on an octadecylsilica column [Capcell pack $\mathrm{C}_{18}$ UG80 column, $20 \times 250 \mathrm{~mm}$ (Shiseido); mobile phase, acetonitrile and isopropyl alcohol ( $1: 1 \mathrm{vol} / \mathrm{vol})$; temperature, $35^{\circ} \mathrm{C}$; and flow rate, $9.9 \mathrm{~mL} /$ min]. Purified MK-9 (4H) was ascertained as a single spot on a thin-layer chromatography plate (silica gel 60 F254 plate; Merck, Darmstadt, Germany). In addition, the purity of the MK-9 $(4 \mathrm{H})$ sample was determined by HPLC with the octadecylsilica column [Capcell pack $\mathrm{C}_{18} \mathrm{SG} 120$ column, $4.6 \times 250 \mathrm{~mm}$; mobile phase, methyl alcohol and ethyl alcohol (1:1 vol/vol); temperature, $40^{\circ} \mathrm{C}$; and flow rate, $1.0 \mathrm{~mL} / \mathrm{min}$ ] and monitoring at $254 \mathrm{~nm}$. The UV and mass spectra were measured with a Shimadzu UV-1600 spectrophotometer and a mass spectrometer (JMS-AM II; Japan Electron Optics, Tokyo, Japan), respectively.

\section{Extraction of Vitamin K from Cheese}

Vitamin $\mathrm{K}$ in the cheese was extracted according to the method of Kojima et al. (2004). Briefly, $2.5 \mathrm{~g}$ of cheese was homogenized with $45 \mathrm{~mL}$ of distilled water, followed by addition of $100 \mathrm{~mL}$ of phosphate buffer $(\mathrm{pH}$ 7.7) and $1.25 \mathrm{~g}$ of lipase. The sample solution was incubated at $37^{\circ} \mathrm{C}$ for $3 \mathrm{~h}$ while stirring with a magnetic stirrer. The hydrolysate was transferred to a $500-\mathrm{mL}$ umber separatory funnel containing $2.5 \mathrm{~mL}$ of $10 \mathrm{~N}$ $\mathrm{NaOH}$ and $100 \mathrm{~mL}$ of $99.5 \%$ ethanol. Vitamin $\mathrm{K}$ was extracted with three $100-\mathrm{mL}$ portions of $n$-hexane. The resultant hexane layer was collected and subsequently evaporated. The dried residue was dissolved in $10 \mathrm{~mL}$ of $n$-hexane and applied on a Sep-Pak silica cartridge (Nihon Waters Co. Ltd., Tokyo, Japan). The quinones were eluted with $15 \mathrm{~mL}$ of $8 \%$ ( $\mathrm{vol} / \mathrm{vol}$ ) diethyl ether in $n$-hexane. The eluate was evaporated and redissolved by adding a small amount of acetone. A small aliquot of this solution was filtered and used for vitamin $\mathrm{K}$ analysis with HPLC. The measurement was performed twice independently.

\section{Analytical HPLC of MK-9 (4H)}

Vitamin K was determined with an HPLC system (Kojima et al., 2004). The HPLC instrument was equipped with a postcolumn electrochemical reducer (Environmental Science Associates, Chelmsford, MA) and a fluorescence detector [FP-1520S (Jasco Co., Tokyo, Japan); Capcell pack $\mathrm{C}_{18}$ SG 120 column, $4.6 \times$ $250 \mathrm{~mm}$ (Shiseido, Kyoto, Japan); mobile phase, methyl alcohol and ethyl alcohol (6:4 vol/vol), with $\mathrm{NaClO}_{4}(7$ $\mathrm{g} / \mathrm{L}$ ); temperature, $45^{\circ} \mathrm{C}$; flow rate, $1.0 \mathrm{~mL} / \mathrm{min}$; and injection volume, $10 \mu \mathrm{L}$ ]. Detection was carried out at an excitation wavelength of $240 \mathrm{~nm}$ and an emission wavelength of $430 \mathrm{~nm}$. Vitamin K concentration was calculated by the external standard method. The repeatability of MK-9 (4H) measurement was determined in Norwegian Jarlsberg cheese. In the present study, the recovery of MK-9 $(4 \mathrm{H})$ was not determined; however, Kojima et al. (2004) reported that the average recovery of MK-9 from milk samples was approximately $85 \%$ or more.

\section{Statistical Methods}

A correlation analysis was performed to examine whether a biomass of propionibacteria was associated with MK-9 (4H) concentration. Spearman's rank correlation was used for the correlation analysis.

\section{RESULTS}

\section{MK-9 (4H) Standard}

We previously found that $P$. freudenreichii ET-3 produced a large amount of MK-9 $(4 \mathrm{H})$ (Furuichi et al., 2006); therefore, the MK-9 $(4 \mathrm{H})$ standard was purified from $P$. freudenreichii. The purified menaquinone sample showed a single spot on the thin-layer chromatography plate, and its UV spectrum showed 4 sharp absorption peaks at 243.0, 248.5, 260.5, and $269.5 \mathrm{~nm}$. The mass spectrum of the purified sample indicated the molecular weight at $\mathrm{m} / z 788$ and fragmented peaks at $\mathrm{m} / \mathrm{z} 719,651,584,516,450,380$, and 227 (Figure 1). These spectra were almost the same as those for MK$9(4 \mathrm{H})$ previously reported by Schwartz (1973). We checked the purity of this sample by a conventional method with HPLC. The chromatogram of this sample indicated a single large peak whose area was more than $95 \%$ that of all the peaks on the chromatogram. Therefore, we used this sample as the standard for HPLC analysis.

\section{Bacterial Counts and Short-Chain Fatty Acid Concentrations in Cheeses}

Because MK-9 $(4 \mathrm{H})$ is produced by propionibacteria and not lactic acid bacteria, we assessed whether the cheeses used in this study were fermented by propionibacteria. Table 2 shows the bacterial counts in the tested cheeses. The viable propionibacterial cell counts were higher in the Swiss Emmental E-1 and Norwegian Jarlsberg J-1 samples than in the other cheese samples. Moreover, the E-1 and J-1 cheese samples contained a large amount of propionate (Table 3 ). In contrast, both propionibacteria cell counts and propionate concentrations in Swiss Appenzeller and Gruyère cheese samples were low. With regard to propionate concentration, the 


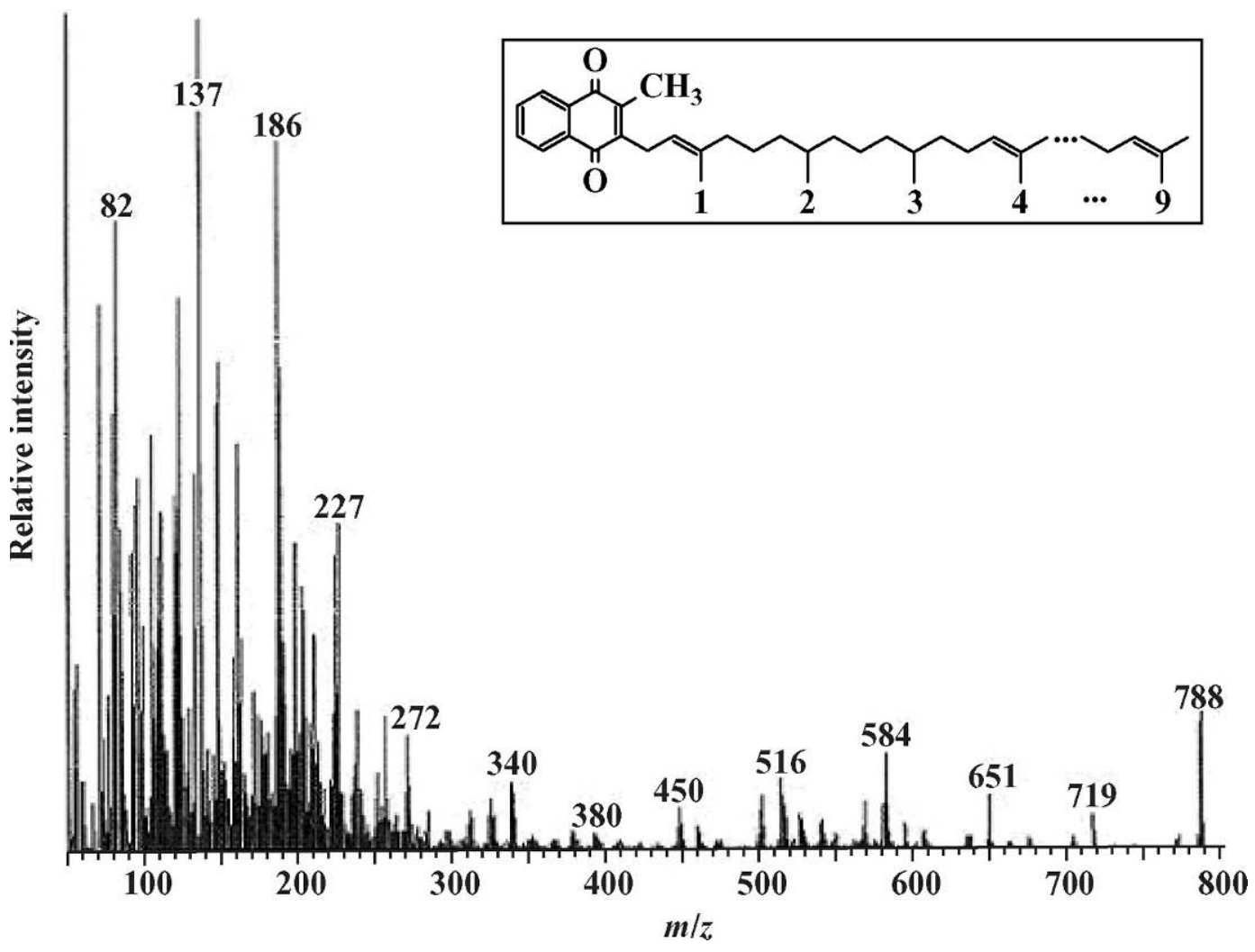

Figure 1. Electron impact mass spectra and chemical structure of tetrahydromenaquinone-9.

values in the French Comte and Swiss Raclette cheese samples appeared to be moderate when compared with the values of all the other cheese samples. In the present study, the propionibacteria cell count and propionate concentration in the cheese samples exhibited a positive correlation $\left(\mathrm{r}^{2}=0.60, P=0.07\right)$.

Lactic acid bacteria were detected in all the cheese samples. However, the viable cell counts of lactoccoci were very low in almost all samples except Appenzeller A-2 and Raclette. Therefore, it is probable that the lac-

Table 2. Bacterial counts in cheese

\begin{tabular}{lcccc}
\hline & \multicolumn{4}{c}{ Viable cell counts (cfu/g) } \\
\cline { 2 - 5 } Sample & Total & & \\
ID & count & Lactococci & Lactobacilli & Propionibacteria \\
\hline A-1 & $1.8 \times 10^{6}$ & $3.0 \times 10^{5}$ & $1.3 \times 10^{6}$ & $1.5 \times 10^{5}$ \\
A-2 & $5.3 \times 10^{7}$ & $5.2 \times 10^{7}$ & $1.4 \times 10^{5}$ & $1.3 \times 10^{6}$ \\
C-1 & $4.1 \times 10^{6}$ & $<10^{3}$ & $3.8 \times 10^{6}$ & $3.0 \times 10^{5}$ \\
C-2 & $2.0 \times 10^{6}$ & $<10^{3}$ & $9.0 \times 10^{5}$ & $1.1 \times 10^{6}$ \\
E-1 & $9.4 \times 10^{7}$ & $1.5 \times 10^{4}$ & $6.9 \times 10^{7}$ & $2.5 \times 10^{7}$ \\
E-2 & $2.6 \times 10^{6}$ & $<10^{3}$ & $2.6 \times 10^{6}$ & $<10^{5}$ \\
G-1 & $7.8 \times 10^{7}$ & $4.0 \times 10^{3}$ & $7.8 \times 10^{7}$ & $4.0 \times 10^{5}$ \\
G-2 & $3.0 \times 10^{6}$ & $<10^{3}$ & $3.0 \times 10^{6}$ & $<10^{5}$ \\
J-1 & $7.5 \times 10^{7}$ & $4.5 \times 10^{4}$ & $3.2 \times 10^{5}$ & $7.5 \times 10^{7}$ \\
R-1 & $7.8 \times 10^{8}$ & $5.7 \times 10^{8}$ & $2.1 \times 10^{8}$ & $2.0 \times 10^{5}$ \\
\hline
\end{tabular}

tococci died and the cell counts largely decreased during cheese ripening and storage.

\section{MK-9 (4H) Concentration in Cheeses}

In the present study, the detection limit of MK-9 $(4 \mathrm{H})$ was $500 \mathrm{pg}$, and the coefficient of variation for the peak area of MK-9 (4H) in Jarlsberg cheese among triplicate analyses was $1.1 \%$. Table 4 shows the concentrations of phylloquinone, MK-4, and MK-9 $(4 \mathrm{H})$ in the cheese samples. Among the cheese samples tested, Jarlsberg

Table 3. Short-chain fatty acids in cheese

\begin{tabular}{lcccc}
\hline & \multicolumn{4}{c}{ Short-chain fatty acid (mmol/g) } \\
\cline { 2 - 5 } ID & Succinate & Lactate & Acetate & Propionate \\
\hline A-1 & 3.7 & 42.2 & 24.6 & 4.0 \\
A-2 & 0.8 & 48.0 & 11.0 & 6.5 \\
C-1 & 5.1 & 139.2 & 29.6 & 23.7 \\
C-2 & 19.1 & 62.8 & 43.3 & 45.2 \\
E-1 & 7.1 & 27.2 & 59.5 & 89.7 \\
E-2 & 2.4 & 61.2 & 49.3 & 31.8 \\
G-1 & 1.4 & 113.5 & 12.3 & 11.2 \\
G-2 & 0.8 & 113.5 & 10.0 & 1.0 \\
J-1 & 7.1 & 0.5 & 53.4 & 106.3 \\
R-1 & 5.8 & 24.5 & 29.0 & 24.7 \\
\hline
\end{tabular}


Table 4. Concentrations of phylloquinone $\left(\mathrm{VK}_{1}\right)$, menaquinone-4 (MK-4), and tetrahydromenaquinone-9 [(MK-9 (4H)] in cheese

\begin{tabular}{llccc}
\hline & \multicolumn{4}{c}{ Vitamin K concentration $(\mathrm{ng} / \mathrm{g})$} \\
\cline { 2 - 5 } ID & VK $_{1}$ & MK-4 & MK-9 (4H) & Total \\
\hline A-1 & 58 & 43 & ND $^{1}$ & 101 \\
A-2 & 47 & 52 & 20 & 119 \\
C-1 & 51 & 84 & 52 & 187 \\
C-2 & 33 & 55 & 60 & 148 \\
E-1 & 52 & 81 & 314 & 447 \\
E-2 & 51 & 86 & 222 & 359 \\
G-1 & 58 & 81 & ND & 139 \\
G-2 & 33 & 96 & ND & 129 \\
J-1 & 60 & 84 & 652 & 796 \\
R-1 & 26 & 50 & 47 & 123 \\
\hline
\end{tabular}

${ }^{1} \mathrm{ND}=$ not detected.

cheese contained the largest amount of MK-9 (4H) $(652$ $\mathrm{ng} / \mathrm{g}$ ). Moreover, the analytical HPLC chromatogram showed the existence of presumptive MK- 8 and MK-9 peaks (Figure 2). We assumed, based on these results, that Jarlsberg cheese contained a large amount of vitamin K. In contrast, Emmental cheeses E-1 and E-2 contained a large amount of MK-9 $(4 \mathrm{H})$ and only small amounts of phylloquinone and MK-4 (Table 4), and the presumptive MK- 8 and MK-9 peaks for these cheeses were not detected. In a previous investigation, MK-8 and MK-9 were not detected in Emmental-type cheeses (Koivu-Tikkanen et al., 2000). Therefore, it is probable

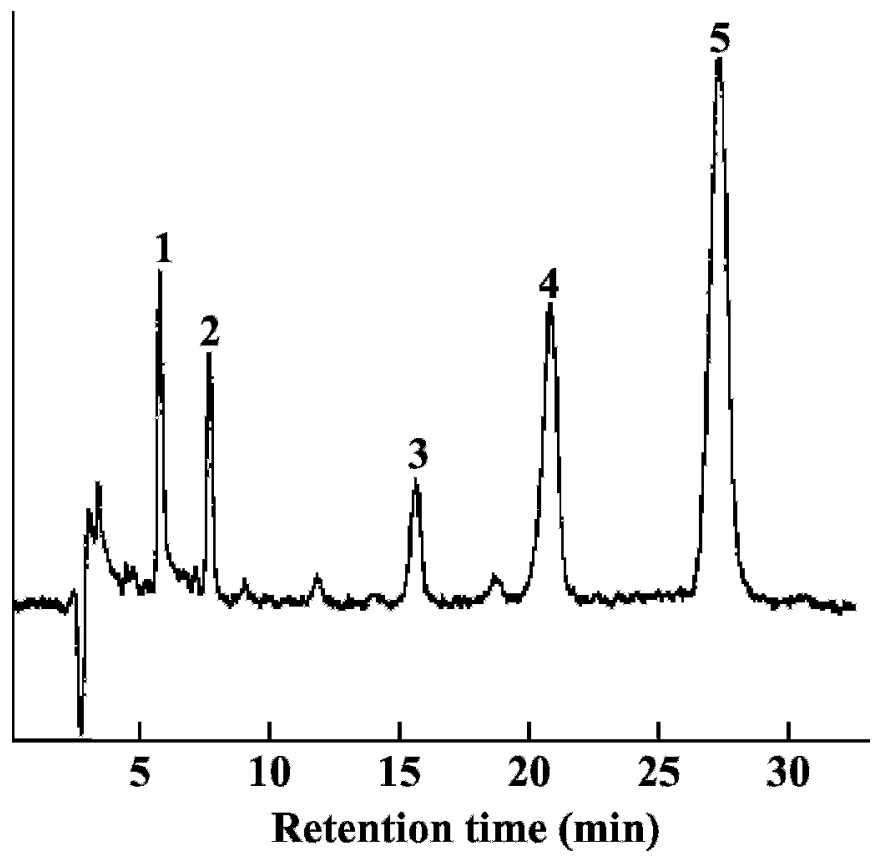

Figure 2. Analytical HPLC chromatogram of Norwegian Jarlsberg cheese. Peaks: 1 , menaquinone (MK)-4; 2 , phylloquinone; 3 , presumptive MK-8; 4, presumptive MK-9; 5, tetrahydromenaquinone-9 [MK$9(4 \mathrm{H})]$. that the major menaquinone in Emmental cheese is MK-9 (4H). In the present study, MK-9 (4H) concentrations were lower in the Comte and Raclette cheeses than in the Jarlsberg and Emmental cheeses. In the Appenzeller A-1 and Gruyère cheeses, MK-9 $(4 \mathrm{H})$ was not detected.

In the present study, the MK-9 $(4 \mathrm{H})$ concentration in the cheese demonstrated a positive correlation with the propionibacteria cell count and propionate concentration $(\mathrm{r}=0.53, P=0.11 ; \mathrm{r}=0.94, P=0.005$, respectively).

\section{DISCUSSION}

In the present study, we found large amounts of MK$9(4 \mathrm{H})$ in a few cheeses. Among the cheeses tested, the MK-9 (4H) concentration was higher in Swiss Emmental and Norwegian Jarlsberg cheeses than in the other cheeses (Table 4). Interestingly, MK-9 (4H) appears to be a major menaquinone in Jarlsberg and Emmental cheeses. Several studies on vitamin K determination in food have been carried out, and MK- 8 and MK-9 have been considered the major vitamin $\mathrm{K}$ types in cheese (Koivu-Tikkanen et al., 2000; Schurgers and Vermeer, 2000). This is because the common starter Lc. lactis produces these menaquinones. Koivu-Tikkanen et al. (2000) reported that in Edam-type cheese, the concentration of vitamin $\mathrm{K}$, which was almost the sum of MK8 ( 100 ng/g) and MK-9 ( 300 ng/g), was $494 \mathrm{ng} / \mathrm{g}$. According to their results, the vitamin $\mathrm{K}$ concentrations in Emmental samples ranged from 80 to $90 \mathrm{ng} / \mathrm{g}$. In our study, the vitamin $\mathrm{K}$ concentrations, that is, the total concentrations of phylloquinone, MK-4, and MK$9(4 \mathrm{H})$, in the Emmental E-1 and E-2 cheese samples were 447 and $359 \mathrm{ng} / \mathrm{g}$, respectively (Table 4). More surprisingly, the Jarlsberg cheese sample contained 60 $\mathrm{ng} / \mathrm{g}$ of phylloquinone, $84 \mathrm{ng} / \mathrm{g}$ of MK-4, and $652 \mathrm{ng} / \mathrm{g}$ of MK-9 (4H) (Table 4); moreover, presumptive MK-8 and MK-9 peaks were observed on the chromatogram (Figure 2). It is probable, based on these results, that the vitamin $\mathrm{K}$ concentrations in these natural cheeses are currently underestimated.

In the present study, vitamin $\mathrm{K}$ concentrations in Emmental and Jarlsberg cheeses were moderate when compared with those in green vegetables $(>1,000 \mathrm{ng} /$ $\mathrm{g}$ of phylloquinone). However, according to a previous report (Schurgers and Vermeer, 2000), phylloquinone absorption from vegetables is very poor (5 to $15 \%$ ), whereas menaquinone (MK-4, MK-7, MK-8, and MK9) absorption from dairy products and the Japanese food natto is considerably better (probably almost complete). Consequently, we can presume that Emmental and Jarlsberg cheeses contain meaningful amounts of vitamin $\mathrm{K}$ because of their high MK-9 (4H) concentra- 
tions. To our knowledge, the MK-9 $(4 \mathrm{H})$ concentration in cheese has not been reported previously; therefore, this paper is the first to describe the quantitative determination of MK-9 $(4 \mathrm{H})$ in cheese.

In this study, the MK-9 $(4 \mathrm{H})$ concentration in cheese showed a correlation with the viable propionibacterial cell count $(\mathrm{r}=0.53, P=0.11)$ and the propionate concentration $(\mathrm{r}=0.94, P=0.005)$. In fact, the MK-9 $(4 \mathrm{H})$ concentration in the Jarlsberg cheese sample, which showed the highest propionibacteria cell count and propionate concentration, was the highest among all the cheese samples. In contrast, both propionibacteria and MK-9 (4H) were not detected in Gruyère cheese G-2. Therefore, from these results it is evident that the increase in propionibacteria in cheese contributed to the generation of MK-9 $(4 \mathrm{H})$.

During cheese ripening and storage, the number of propionibacteria decreases with cell death and subsequent autolysis (Bouton et al., 1998; Treimo et al., 2006). Furuichi et al. (2006) suggested that $P$. freudenreichii ET-3 cells accumulate MK-9 $(4 \mathrm{H})$ during the growth period and release it after their lysis. Although cheese generally has a long expiration date, information about the stability of vitamin $\mathrm{K}$ in dairy products is far from complete. Accordingly, further studies are required to examine whether MK-9 $(4 \mathrm{H})$ is stable in cheese stored for a long time.

Previously, it was believed that vitamin K affected only blood coagulation (Shearer, 1995). A further spur to vitamin $\mathrm{K}$ research has been the discovery of a diverse group of vitamin $\mathrm{K}$-dependent proteins that are unrelated to blood coagulation but implicated in calcium homeostasis (Kaneki et al., 2006). According to several articles (Iwamoto et al., 2004; Kaneki et al., 2006), vitamin K deficiency may be associated with low bone mineral density and increased risk of bone fracture. Therefore, vitamin $\mathrm{K}$ has emerged as a potential protector against osteoporosis. We presumed, based on the present study, that various types of natural cheeses may contain meaningful amounts of vitamin $\mathrm{K}$ because of the presence of menaquinones derived from the bacterial species grown in cheese. Moreover, dairy products, including cheese, generally contain abundant calcium. Therefore, we believe that the consumption of natural cheese would contribute to human bone health.

In conclusion, the results in this study suggest that the quantity of vitamin K in Swiss Emmental and Norwegian Jarlsberg cheeses is greater than that estimated conventionally, and that the main constituent of vita- min $\mathrm{K}$ in cheese is MK-9 $(4 \mathrm{H})$. We believe that several natural cheeses may become candidate dairy products for the prevention of osteoporosis because of their abundant menaquinone and calcium contents.

\section{ACKNOWLEDGMENTS}

The authors are extremely grateful to Shiro Kawabata for his valuable discussions and Saori Murata for providing technical assistance. We also thank Naoki Orii for providing encouragement throughout the study.

\section{REFERENCES}

Bouton, Y., P. Guyot, and R. Grappin. 1998. Preliminary characterization of microflora of Comte cheese. J. Appl. Microbiol. 85:123-131.

Britz, T. J., and K. H. Riedel. 1994. Propionibacterium species diversity in Leerdammer cheese. Int. J. Food Microbiol. 22:257-267.

Furuichi, K., K. Hojo, Y. Katakura, K. Ninomiya, and S. Shioya. 2006. Aerobic culture of Propionibacterium freudenreichii ET-3 can increase production ratio of 1,4-dihydroxy-2-naphthoic acid to menaquinone. J. Biosci. Bioeng. 101:464-470.

Iwamoto, J., T. Takeda, and Y. Sato. 2004. Effects of vitamin $\mathrm{K}_{2}$ on osteoporosis. Curr. Pharm. Des. 10:2557-2576.

Kaneki, M., T. Hosoi, Y. Ouchi, and H. Orimo. 2006. Pleiotropic actions of vitamin K: Protector of bone health and beyond? Nutrition 22:845-852.

Koivu-Tikkanen, T. J., V. Ollilainen, and V. I. Piironen. 2000. Determination of phylloquinone and menaquinones in animal products with fluorescence detection after postcolumn reduction with metallic zinc. J. Agric. Food Chem. 48:6325-6331.

Kojima, T., M. Asoh, N. Yamawaki, T. Kanno, H. Hasegawa, and A. Yonekubo. 2004. Vitamin K concentrations in the maternal milk of Japanese women. Acta Paediatr. 93:457-463.

Kure, C. F., and I. Skaar. 2000. Mould growth on the Norwegian semi-hard cheeses Norvegia and Jarlsberg. Int. J. Food Microbiol. 62:133-137.

Morishita, T., N. Tamura, T. Makino, and S. Kudo. 1999. Production of menaquinones by lactic acid bacteria. J. Dairy Sci. 82:1897-1903.

Niwa, T., M. Nakao, S. Hoshi, K. Yamada, K. Inagaki, M. Nishida, and T. Nabeshima. 2002. Effect of dietary fiber on morphineinduced constipation in rats. Biosci. Biotechnol. Biochem. 66:1233-1240.

Purves, E. 2005. Neonatal hematologic disorders. J. Pediatr. Oncol. Nurs. 22:168-175.

Schurgers, L. J., and C. Vermeer. 2000. Determination of phylloquinone and menaquinones in food. Effect of food matrix on circulating vitamin $\mathrm{K}$ concentrations. Haemostasis 30:298-307.

Schwartz, A. C. 1973. Terpenoid quinones of the anaerobic Propionibacterium shermanii I. (II, III)-Tetrahydromenaquinone-9. Arch. Mikrobiol. 91:273-279.

Shearer, M. J. 1990. Vitamin K and vitamin K-dependent proteins. Br. J. Haematol. 75:156-162.

Shearer, M. J. 1995. Vitamin K. Lancet 345:229-234.

Thierry, A., M. B. Maillard, R. Richoux, J. R. Kerjean, and S. Lortal. 2005. Propionibacterium freudenreichii strains quantitatively affect production of volatile compounds in Swiss cheese. Lait 85:57-74.

Treimo, J., G. Vegarud, T. Langsrud, and K. Rudi. 2006. Use of DNA quantification to measure growth and autolysis of Lactococcus and Propionibacterium spp. in mixed populations. Appl. Environ. Microbiol. 72:6174-6182. 